\title{
Cancer patterns and association with mortality and renal outcomes in non- dialysis dependent chronic kidney disease: a matched cohort study
}

\author{
Rajkumar Chinnadurai ${ }^{1,2^{*}}$ (D, Emma Flanagan ${ }^{3}$, Gordon C. Jayson ${ }^{4,5}$ and Philip A. Kalra ${ }^{1,2}$
}

\begin{abstract}
Background: Cancer in patients with chronic kidney disease (CKD) is an added burden to their overall morbidity and mortality. Cancer can be a cause or an effect of CKD. In CKD patients, a better understanding of cancer distribution and associations can aid in the proper planning of renal replacement therapy (RRT) and in the choice of chemotherapeutic agents, many of which are precluded in more advanced CKD. This study aims to investigate the distribution and the association of cancer with mortality, renal progression and RRT assignment in a non-dialysis dependent CKD cohort, few studies have investigated this in the past.

Methods: The study was carried out on 2952 patients registered in the Salford Kidney Study (SKS) between October 2002 and December 2016. A comparative analysis was performed between 339 patients with a history of cancer (previous and current) and 2613 patients without cancer at recruitment. A propensity score matched cohort of 337 patients was derived from each group and used for analysis. Cox-regression models and Kaplan-Meier estimates were used to compare the association of cancer with mortality and end-stage renal disease (ESRD) outcomes. Linear regression analysis was applied to generate the annual rate of decline in estimated glomerular filtration rate (delta eGFR).

Results: Of our cohort, 13.3\% had a history of cancer at recruitment and the annual rate of de novo cancers in the non-cancer patients was 1.6\%. Urogenital cancers including kidney and bladder, and prostate and testicle in males, ovary and uterus in females, were the most prevalent cancers (46\%), as expected from the anatomical or physiological roles of these organs and relationship to nephrology. Over a median follow-up of 48 months, 1084 (36.7\%) of patients died. All-cause mortality was higher in the previous and current cancer group (49.6\% vs 35\%, $p<0.001$ ), primarily because of cancer-specific mortality. Multivariate Cox regression analysis showed a strong association of cancer with all-cause mortality (HR:1.41;95\%Cl: 1.12-1.78; $p=0.004)$. There was no difference between the groups regarding reaching end-stage renal disease (26\% in both groups) or the rate of decline in eGFR ( -0.97 for cancer vs $-0.93 \mathrm{~mL} / \mathrm{min} /$ year for non-cancer, $p=0.93$ ). RRT uptake was similar between the groups $(17.2 \%$ vs $19.3 \%, p=0.49)$.

(Continued on next page)
\end{abstract}

\footnotetext{
*Correspondence: rajkumar.chinnadurai@srft.nhs.uk

'Department of Renal Medicine, Salford Royal NHS Foundation Trust, M6

8HD, Salford, UK

${ }^{2}$ Faculty of Biology, Medicine and Health, University of Manchester,

Manchester, UK

Full list of author information is available at the end of the article
}

(c) The Author(s). 2019 Open Access This article is distributed under the terms of the Creative Commons Attribution 4.0 International License (http://creativecommons.org/licenses/by/4.0/), which permits unrestricted use, distribution, and reproduction in any medium, provided you give appropriate credit to the original author(s) and the source, provide a link to the Creative Commons license, and indicate if changes were made. The Creative Commons Public Domain Dedication waiver (http://creativecommons.org/publicdomain/zero/1.0/) applies to the data made available in this article, unless otherwise stated. 
(Continued from previous page)

Conclusions: Cancer status proved to be an added burden and an independent risk factor for all-cause mortality but not for renal progression. CKD patients with a previous or current history of cancer should be assessed on a case by case basis in planning for renal replacement therapy options, and the presence of cancer should not be a limitation for RRT provision including transplantation.

Keywords: Cancer, Chronic kidney disease (CKD), All-cause mortality, End-stage renal disease, CKD progression, propensity score matching

\section{Introduction}

Cancer is one of the leading causes of mortality and morbidity worldwide [1, 2]. In Europe, it is estimated that there will be 3.91 million new cases and 1.93 million deaths from cancer in 2018 [3]. Cancer prevalence is increasing in chronic kidney disease (CKD) patients due to improvements in life expectancy and better quality of care $[4,5]$. Cancer and CKD are interrelated in many ways. Cancer patients develop CKD on account of the site of cancer, metastases, necessary chemotherapeutic treatments and management of related complications. Despite advances, chemotherapy-induced nephrotoxicity can be a significant barrier in the optimum management of cancer patients, more so in patients with CKD [6].

On the other hand, CKD is a risk factor for developing certain types of cancers such as liver and urogenital tract cancers [7-10]. Also, the presence of CKD in cancer patients is associated with a worse prognosis [11-13]. However, cardiovascular disease is still the leading cause of mortality in CKD patients [14].

In recent years, researchers have shown an increased interest in exploring the associations of cancer in CKD patients, leading to the emergence of the field of onconephrology [15-17]. Several inflammatory and oxidative stress mechanisms have been implicated in linking cancer with CKD $[18,19]$. End-stage renal disease patients on dialysis or after transplantation are high-risk groups identified for cancer development due to uremia and immunodeficiency [20-23]. But, research on cancer and its impact on mortality and renal outcome is scarce in a Caucasian population with advanced CKD (CKD-3-5, not on dialysis) and the question of whether the presence of cancer accelerates the rate of progression of CKD is unexplored, which this study aims to address.

\section{Materials and methods \\ Sampling}

We conducted this study in the Salford Kidney Study (SKS) patients enrolled between October 2002 until December 2016. SKS, previously known as Chronic Renal Insufficiency Standard Implementation Study (CRISIS), is a large prospective cohort study recruiting non-dialysis CKD patients since 2002. Patient recruitment in the SKS has been described in previous published studies [24, 25]. In brief, any non-dialysis CKD patient above the age of 18 years and an eGFR less than $60 \mathrm{~mL} / \mathrm{min} / 1.73 \mathrm{~m}^{2}$ referred to our tertiary renal service (1.55 million catchment population) is eligible to participate in this study. Upon recruitment, a questionnaire which includes patient demographic details, comorbidities including the history of malignancy and concurrent medications is completed. The patients are then followed up annually and comorbidities, cardiovascular events and hospital admissions are recorded. All patients provide informed consent, have blood results recorded upon registration and at annual follow up visits. The study has ethical approval for all observational data including mortality outcomes.

From a list of 3115 patients registered in the SKS over this 14-year period, 2952 patients with complete follow-up datasets were eligible. Of these, 392 reported having a history of cancer at study entry. All patients with a history of cancer (current and past) were included irrespective of the cancer site, stage and treatment status to allow calculation of the prevalence and incidence. In patients with multiple cancers, the first developed cancer was taken as the index cancer. Patients with non-melanoma skin (NMS) cancer were included in the no cancer group for further analysis. A matched cohort was generated using propensity score matching and was used for analysis. A flowchart of patient recruitment to the study is shown in Fig. 1.

\section{Data gathering}

The date of SKS entry was used as the study baseline, and all patients were followed until they reached a study endpoint which was one of the following 1) commencement of renal replacement therapy (RRT) 2) death 3) end of analysis period - 31st December 2017 4) lost to follow up.

Data on patient baseline characteristics, baseline blood results, and date of death were gathered from the SKS database and electronic patient records (EPR). Initial blood results were those obtained at study entry or within 3 months. Smoking history was defined as a history of current or previous smoking, irrespective of the number of cigarettes smoked. Similarly, an alcohol history was defined as a history of current or past alcohol intake irrespective of the number of units. Non-fatal cardiovascular events (NFCVE) included a composite of an 
Total patients registered in SKS between October 2002 and December 2016 3115

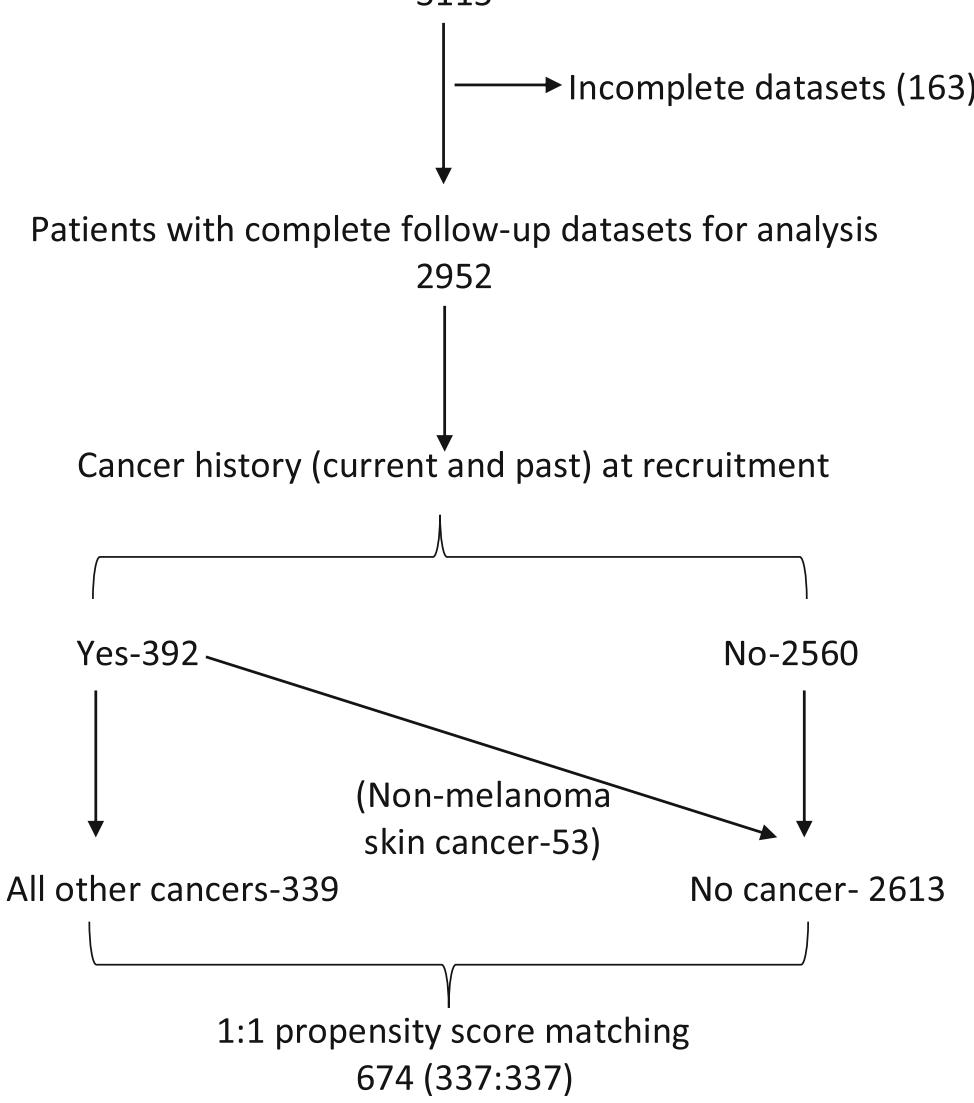

Patients with complete follow-up datasets for analysis

2952

Fig. 1 Flowchart of patient recruitment to the study

acute coronary syndrome, myocardial infarctions, nonfatal cardiac arrest, new diagnosis or hospital admission with congestive cardiac failure, cerebrovascular event and peripheral vascular disease. We defined end-stage renal disease (ESRD) as patients reaching RRT or eGFR $\leq 10 \mathrm{~mL} / \mathrm{min} / 1.73 \mathrm{~m}^{2}$ in patients who opted for supportive care [26]. The cause of death was obtained from death certificates provided by the Office of National Statistics until the end of 2013 and thereafter from the Salford Royal electronic patient record only for those patients who died at our centre before December 2017. The eGFR values were calculated by the CKD epidemiology collaboration (CKD-EPI) formula [27].

\section{Statistical analysis}

Propensity score matching was used to match patients with and without cancer to overcome the effects of selection bias and confounding factors [28]. The matching was undertaken by including all 20 baseline variables: age, gender, ethnicity, smoking status, alcohol consumption, systolic blood pressure (BP), diastolic BP, history of hypertension, diabetes mellitus, ischemic heart disease, myocardial infarction, congestive cardiac failure, cerebrovascular accident, peripheral vascular disease, chronic obstructive pulmonary disease, liver disease, use of statins, erythropoietin, renin-angiotensin blocking agents (ACE inhibitor or angiotensin receptor antagonist) and estimated glomerular filtration rate (eGFR). Matching was undertaken in a 1:1 ratio using the nearest neighbour method with the same propensity score. Comparative analyses of baseline characteristics, baseline blood results, mortality and renal replacement therapy were undertaken on both the total and propensity-matched samples.

Continuous non-parametric variables are presented as median (interquartile range), and the Mann-Whitney $\mathrm{U}$ test was used to compare statistical significance. Categorical data are expressed in percentages, and the Chi-square test was used for comparison.

The association of cancer with mortality and renal outcome (RRT) was calculated using univariate and multivariate Cox proportional hazards models to determine hazard ratios, 95\% confidence intervals and statistical significance. To overcome the influence of competing risks, hazard ratios were derived by censoring at the competing event [29]. The multivariate models were developed by including the covariates based on the clinical plausibility of the causal 
association with outcome. A Kaplan-Meier (KM) curve was used to demonstrate cumulative survival. KM charts were also generated for all-cause mortality and RRT free survival by splitting the cancer group into three subgroups based on their cancer status at baseline; group A: concurrent cancer (diagnosed or treated in the previous 1 year), group B: history of cancer $<5$ years (diagnosed or treated between $>1$ year and $<5$ years) and group $\mathrm{C}$ : cancer $>5$ years (diagnosed or treated $>5$ years ago). The association of cancer with CKD progression was computed using the rate of change of eGFR (eGFR slope) from study entry to study endpoint as assessed by the linear regression slope generated using serial serum creatinine measurements from outpatient clinic visits. Only patients with a minimum of three eGFR measurements were included in this model. The MannWhitney $\mathrm{U}$ test was used to compare the statistical significance between the groups. A $p$-value $<0.05$ was considered statistically significant throughout the analysis. All statistical analysis was performed using IBM SPSS (Version 22), licenced to the University of Manchester. A competing risk analysis (CRA) for RRT, death and incident cancer between the groups was also performed using 'cmprsk' and 'survival' packages in R software, version 3.5.1 [30, 31]. A $p$-value for the CRA was calculated by the modified $\mathrm{X}^{2}$ statistic outlined in Gray, 1988 [32].

\section{Results}

A history of previous or current cancer was evident in $13.3 \%(392 / 2952)$ of our cohort, and the annual rate of de novo cancers in the non-cancer patients was $1.6 \%$. The frequency distribution of various types of cancer is illustrated in Fig. 2. Urological cancers including prostate, kidney, bladder and ureter were the predominant sites of cancer in our cohort, contributing a third of the total number of cancers (32.6\%). Of the 56 patients with skin cancers, 53 had non-melanomatous skin (NMS) cancer and three patients had low risk melanoma. All patients with skin cancer were treated curatively by local excision and had no recurrence during follow up. As NMS cancer patients were included in the no cancer group, the further analysis included 339 patients in the cancer group and 2613 patients in the no cancer group. The propensity score matched sample had 337 patients in each group.

The median age of our total cohort was 67 years with a predominance of males (62\%) and Caucasians 96.1\% (Table 1). Patients with a cancer history were older than those without cancer $(71$ versus 67 years, $p<0.001)$ and had a higher systolic blood pressure (140 versus 138 $\mathrm{mmHg}, p=0.019)$. The groups were similar in distribution in terms of all cardiovascular risk factors at registration apart from the history of hypertension, which was more prevalent in patients without cancer. Other factors that were significantly different between the groups included the history of chronic obstructive pulmonary disease (COPD) and use of renin-angiotensin system (RAS) blockers. On review of initial blood results, the patients in the cancer group had a lower median haemoglobin

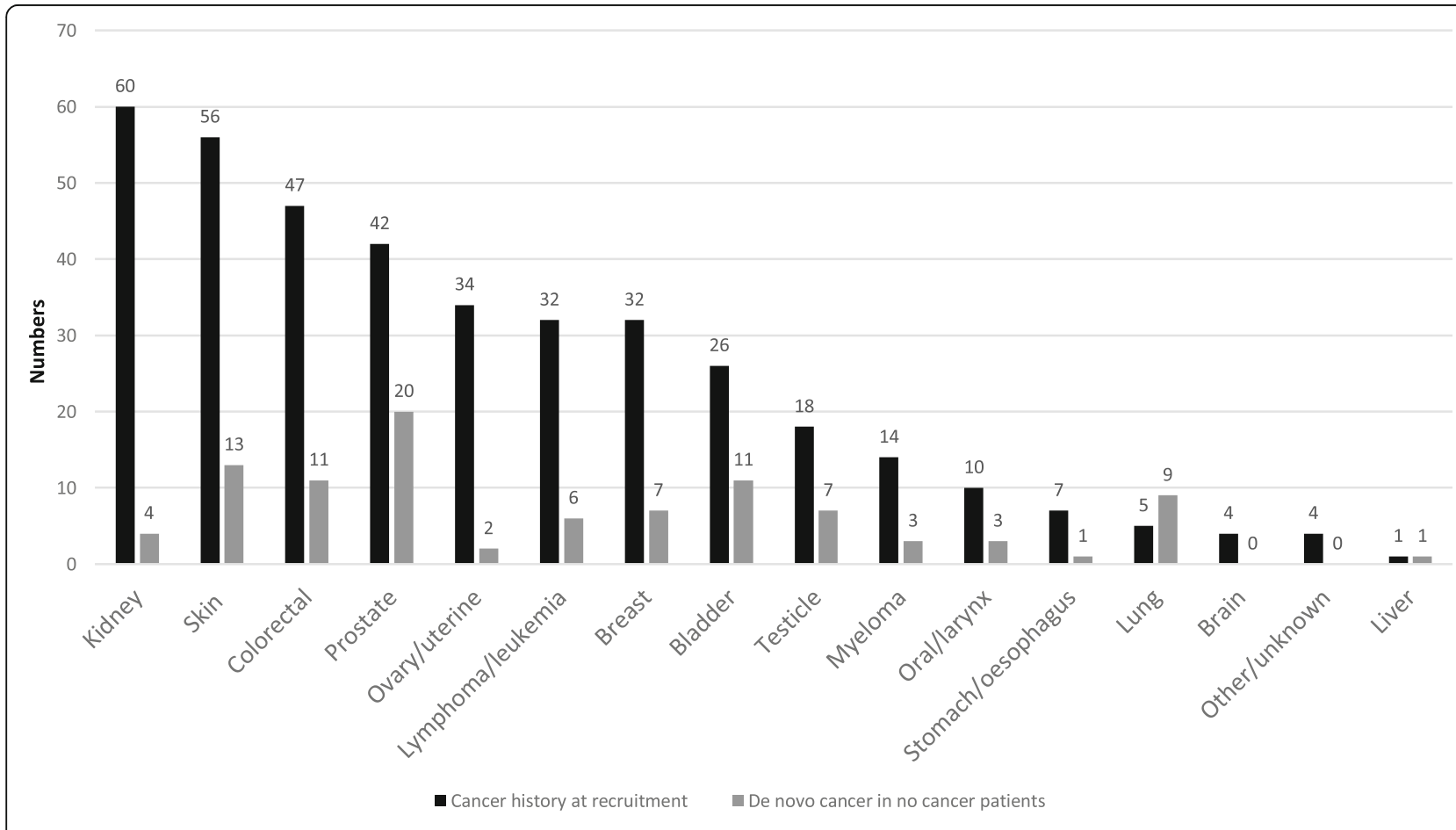

Fig. 2 Cancer site distribution 
Table 1 Comparison of baseline characteristics between cancer and no cancer patients in both total and matched samples

\begin{tabular}{|c|c|c|c|c|c|c|c|c|}
\hline \multirow[b]{2}{*}{ Variable } & \multirow[b]{2}{*}{ Total (2952) } & \multirow{2}{*}{$\begin{array}{l}\text { Total sample } \\
\text { Cancer (339) }\end{array}$} & \multirow[b]{2}{*}{ No cancer (2613) } & \multirow[b]{2}{*}{$p$-Value } & \multicolumn{4}{|c|}{ Propensity matched sample } \\
\hline & & & & & Total (674) & Cancer (337) & No cancer (337) & $p$-Value \\
\hline Age, years & $67(56-76)$ & $71(63-77)$ & $67(55-75)$ & $<0.001$ & $71(63-77)$ & $71(63-77)$ & $71(61-78)$ & 0.79 \\
\hline Male & $1834(62.1 \%)$ & $221(65.2 \%)$ & $1613(61.7 \%)$ & 0.22 & $434(64.4 \%)$ & $220(65.3 \%)$ & $214(63.5 \%)$ & 0.63 \\
\hline Caucasian & 2837 (96.1\%) & 337 (99\%) & $2500(95.67 \%)$ & 0.001 & $670(99.4 \%)$ & $335(99.4 \%)$ & 335 (99.4\%) & 1.00 \\
\hline Smoking & 1941 (65.8\%) & $228(67.3 \%)$ & $1713(65.5 \%)$ & 0.54 & $450(66.8 \%)$ & $227(67.3 \%)$ & $223(66.2 \%)$ & 0.74 \\
\hline Alcohol & 1375 (46.5\%) & $144(43 \%)$ & $1231(47 \%)$ & 0.11 & $289(42.9 \%)$ & $144(42.7 \%)$ & $145(43 \%)$ & 0.94 \\
\hline $\mathrm{BMI}{ }^{\mathrm{a}}, \mathrm{kg} / \mathrm{m}^{2}$ & $28(24-32.6)$ & $28.3(24.7-32.7)$ & $28(24.6-32.6)$ & 0.60 & $28(24.8-31.6)$ & $28.3(24.7-32.8)$ & $27.8(24.8-31)$ & 0.22 \\
\hline Systolic BP, mmHg & $138(124-153)$ & $140(126-156)$ & $138(124-152)$ & 0.019 & 139 (126-155) & $140(126-156)$ & $138(125-152)$ & 0.22 \\
\hline Diastolic BP, mmHg & $75(66-80)$ & $74(66-80)$ & $75(66-80)$ & 0.58 & $74(66-80)$ & $74(66-80)$ & $75(68-80)$ & 0.41 \\
\hline Hypertension & 2684 (90.9\%) & 295 (87\%) & 2389 (91.4\%) & 0.008 & $593(87.9 \%)$ & 295 (87.5\%) & $298(88.4 \%)$ & 0.72 \\
\hline DM & 961 (37.6\%) & $108(31.9 \%)$ & $853(32.6 \%)$ & 0.77 & 215 (31.9\%) & $108(32 \%)$ & 107 (31.7\%) & 0.93 \\
\hline CVD & $1269(43 \%)$ & $151(44.5 \%)$ & 1118 (42.8\%) & 0.54 & $298(44.2 \%)$ & 151 (44.8\%) & 147 (43.6\%) & 0.76 \\
\hline COPD & 545 (18.5\%) & 44 (12.9\%) & $501(23.7 \%)$ & 0.006 & 87 (12.9\%) & 44 (13.1\%) & $43(12.7 \%)$ & 0.91 \\
\hline CLD & 88 (2.9\%) & $7(2.1 \%)$ & 81 (3.1\%) & 0.29 & $13(1.9 \%)$ & 7 (2.1\%) & $6(1.8 \%)$ & 0.78 \\
\hline RAS blocker & 1799 (61\%) & $162(47.7 \%)$ & 1637 (62.6\%) & $<0.001$ & $312(46.3 \%)$ & 162 (48.1\%) & 150 (44.5\%) & 0.35 \\
\hline Statin & 1732 (58.7\%) & $194(57.2 \%)$ & 1538 (59\%) & 0.57 & 391 (58\%) & 194 (57.6\%) & $197(58.5 \%)$ & 0.82 \\
\hline ESA & 379 (12.8\%) & $40(11.8 \%)$ & 339 (13\%) & 0.54 & $73(10.8 \%)$ & $40(11.8 \%)$ & $33(9.8 \%)$ & 0.39 \\
\hline $\mathrm{HB}, \mathrm{g} / \mathrm{L}$ & $123(112-135)$ & $121(109-131)$ & $123(112-135)$ & 0.02 & $122(112-133)$ & $121(110-131)$ & $123(113-134)$ & 0.05 \\
\hline Albumin, g/L & $43(40-45)$ & $43(40-45)$ & $43(40-45)$ & 0.30 & $43(40-45)$ & $43(40-45)$ & $43(40-45)$ & 0.50 \\
\hline ALP, Units/L & $82(66-104)$ & 85.5 (68-107) & $82(65-103)$ & 0.04 & $84(67-107)$ & $85.5(68-107)$ & $85.5(69-108.5)$ & 0.99 \\
\hline Calcium, mmol/L & $2.3(2.2-2.4)$ & $2.32(2.2-2.4)$ & $2.30(2.2-2.4)$ & 0.17 & $2.31(2.2-2.4)$ & $2.32(2.2-2.4)$ & $2.30(2.2-2.4)$ & 0.55 \\
\hline Phosphate, mmol/L & $1.12(0.97-1.3)$ & $1.10(0.95-1.3)$ & $1.12(0.98-1.3)$ & 0.35 & $1.11(0.96-1.3)$ & $1.10(0.95-1.3)$ & $1.12(0.97-1.3)$ & 0.49 \\
\hline uPCR, $\mathrm{g} / \mathrm{mol}$ & $31.5(13-104)$ & $34.23(13.2-87)$ & $31.2(13-107)$ & 0.49 & $33.3(13.1-90.7)$ & $33.7(13.1-85)$ & $32.1(13.5-94.7)$ & 0.64 \\
\hline Creatinine, micromol/L & $182(135-256)$ & $188(141-262)$ & $179(135-254)$ & 0.16 & $185(138-262)$ & $187(141-261)$ & $183(135-263)$ & 0.53 \\
\hline $\mathrm{eGFR}, \mathrm{mL} / \mathrm{min} / 1.73 \mathrm{~m}^{2}$ & $30(19.7-42.5)$ & $28.3(18.3-39.3)$ & $30(19.8-43)$ & 0.02 & $29.3(18.3-40.5)$ & $28.7(18.5-39.4)$ & $29.6(18-40.9)$ & 0.60 \\
\hline
\end{tabular}

a-BMI missing in 530/2952 of total sample and in 124/674 of matched sample. b-missing uPCR values in 292 patients of total sample and 75 patients of matched sample

BMI-body mass index, BP-blood pressure, DM-diabetes mellitus, CVD- cardio vascular disease (includes at least one of the following- ischemic heart disease, myocardial infarction, congestive cardiac failure, cerebrovascular accident, and peripheral vascular disease), COPD-chronic obstructive pulmonary disease, CLDchronic liver disease, RAS-renin-angiotensin system blocker, ESA-erythropoietin stimulating agents, HB-haemoglobin, ALP-alkaline phosphatase, UPCR-urine protein creatinine ratio, eGFR - estimated glomerular filtration rate calculated by CKD-EPI equation

Continuous variables are expressed as median (interquartile range) and $p$-Value by Man-Whitney $\mathrm{U}$ test

Categorical variables are expressed as number (\%) and $p$-Value by Chi-Square test. Statistically significant $p$-values are displayed in bold (i.e. $p<0.05$ )

(121 versus $123 \mathrm{~g} / \mathrm{l}, p=0.02)$, and eGFR (28.3 versus 30 $\mathrm{mL} / \mathrm{min} / 1.73 \mathrm{~m}^{2}, p=0.018$; Table 1). Although these differences noted are statistically different, they are probably not clinically significant. After propensity score matching the groups were very closely matched with no difference noted between the groups in any of the registration or biochemical characteristics.

During a median follow up of 48 months, 1084 (36.7\%) patients died. The all-cause mortality rate was significantly higher in the cancer group even in the matched groups (49.3\% versus $38.3 \%, p=0.004)$. The cause of death data was available only in 474 of the 1084 patients who died (44\%). Cancer-related death was significantly higher in the cancer group than the no cancer group $(26.3 \%$ versus $13.5 \%, p=0.005)$. Deaths due to cardiovascular disease and infections were similar in the groups. In the matched sample, age at death was significantly less in patients with cancer ( 79 versus 80.5 years, $p=0.03$ ) (Table 2 ). This survival difference is also illustrated in the KM survival curve (log-rank; $p=0.002$ ) (Fig. 3).

There were a total of 282 NFCVE reported during the follow-up period and the events were similar between the groups in both the overall and propensity-matched analyses. On review of renal outcomes, 30\% of patients reached ESRD. The median age of the patients at the time of commencement of haemodialysis was 63 years, peritoneal dialysis was 62 years, and a transplant was 52 years. The RRT uptake was similar in the two groups in the matched sample $(17.2 \%$ versus $19.3 \%, p=0.49)$, also shown in KM curve (log-rank; $p=0.93$; Fig. 3 ). The first RRT modality was predominantly haemodialysis in the cancer group (68.9\%). More patients in the no cancer group received a 
Table 2 Comparison of mortality and renal outcomes between cancer and no cancer patients in both total and matched sample

\begin{tabular}{|c|c|c|c|c|c|c|c|c|}
\hline \multirow[b]{2}{*}{ Variable } & \multirow[b]{2}{*}{ Total (2952) } & \multicolumn{3}{|l|}{ Total sample } & \multirow[b]{2}{*}{ Total (674) } & \multirow{2}{*}{$\begin{array}{l}\text { Propensity } \\
\text { Cancer (337) }\end{array}$} & \multicolumn{2}{|l|}{ matched sample } \\
\hline & & Cancer (339) & No cancer (2613) & $p$-Value & & & No cancer (337) & $p$-Valu \\
\hline ollow-up, months & $48(25-79)$ & $40.3(20.5-68)$ & $48.2(25-79)$ & 0.001 & $43(21-75)$ & $40.6(21-68)$ & $47.1(21.6-78.5)$ & 0.09 \\
\hline Age at death, years & $78.7(72-84)$ & $79(72.4-84.7)$ & $78.5(72.1-84.4)$ & 0.88 & $79(72-84)$ & $79(72-84)$ & $80.5(75-86)$ & 0.03 \\
\hline All-cause mortality & $1084(36.7 \%)$ & $168(49.6 \%)$ & $916(35 \%)$ & $<0.001$ & $295(43.8 \%)$ & $166(49.3 \%)$ & $129(38.3 \%)$ & 0.004 \\
\hline Cause of death (cancer) & $74 / 474(15.6 \%)$ & 20/76 (26.3\%) & $54 / 398(13.5 \%)$ & 0.005 & 26/128 (20.3\%) & $20 / 76(26.3 \%)$ & $6 / 52(11.5 \%)$ & 0.04 \\
\hline Cause of death (CVD) & $154 / 474(32.5 \%)$ & 20/76 (26.3\%) & 134/398 (33.7\%) & 0.21 & $33 / 128(25.7 \%)$ & 20/76 (26.3\%) & $13 / 52(25 \%)$ & 0.87 \\
\hline ath (infection) & $124 / 474(26.1 \%)$ & $16 / 76(21 \%)$ & 108/398 (27.1\%) & 0.27 & $30 / 128(23.4 \%)$ & 16/76 (21\%) & $14 / 52(27 \%)$ & 0.44 \\
\hline NFCVE & $282(9.55 \%)$ & $30(8.9 \%)$ & $252(9.6 \%)$ & 0.64 & $52(7.71 \%)$ & $30(8.9 \%)$ & $22(6.5 \%)$ & 0.25 \\
\hline ESRD $(\mathrm{RRT}+\mathrm{eGFR}<\mathrm{or}=10)$ & $855(29.9 \%)$ & 87 (25.7\%) & $768(29.4 \%)$ & 0.16 & $174(25.8 \%)$ & $87(25.8 \%)$ & 87 (25.8\%) & 1.00 \\
\hline$\sigma$ & $233(7.9 \%)$ & $29(8.6 \%)$ & $204(7.8 \%)$ & 0.18 & $51(7.6 \%)$ & $29(8.6 \%)$ & $22(6.5 \%)$ & 0.24 \\
\hline RRT & $622(21 \%)$ & $58(17.1 \%)$ & $564(21.6 \%)$ & 0.06 & $123(18.25 \%)$ & $58(17.2 \%)$ & $65(19.3 \%)$ & 0.49 \\
\hline First start RRT modality (TX) & $97 / 622(15.5 \%)$ & $3 / 58(5.17 \%)$ & $94 / 564(16.6 \%)$ & 0.02 & 10/123 (8.1\%) & $3 / 58(5.2 \%)$ & $7 / 65(10.7 \%)$ & 0.26 \\
\hline First start RRT modality (HD) & $323 / 622(51.9 \%)$ & $40 / 58(68.9 \%)$ & $283 / 564(50.2 \%)$ & 0.006 & $78 / 123(63.4 \%)$ & $40 / 58(68.9 \%)$ & $38 / 65(58.5 \%)$ & 0.23 \\
\hline First start RRT modality (PD) & $202 / 622(32.5 \%)$ & $15 / 58(25.9 \%)$ & $187 / 564(33.2 \%)$ & 0.26 & $35 / 123(28.5 \%)$ & $15 / 58(25.8 \%)$ & $20 / 65(30.8 \%)$ & 0.55 \\
\hline
\end{tabular}

Cause of death represents 1a cause of death in death certificate. Cause of death available only in 474/1084 patients of the total sample (76/168 in cancer group and 398/916 in no cancer group) and 128/295 of matched sample (76/166 in cancer group and 52/129 in no cancer group). CVD-cardiovascular disease NFCVE non-fatal cardiovascular events, ESRD end-stage renal disease, eGFR estimated glomerular filtration rate, $R R T$ renal replacement therapy, CC conservative care, $T X$ renal transplant, $P D$ peritoneal dialysis, $H D$ haemodialysis. Continuous variables are expressed as median (interquartile range) and $p$-Value by ManWhitney U test

Categorical variables are expressed as number (\%) and $p$-Value by Chi-square test. Statistically significant $\mathrm{p}$-values are displayed in bold (i.e. $p<0.05$ )

renal transplant compared to the cancer group $(42.9 \%$ versus $15.5 \%, p<0.001)$, but these differences were not observed once the groups were matched (Table 2).

In Cox-proportional hazard models, a cancer history at baseline showed strong association with all-cause mortality in the univariate model (HR: 1.64; 95\%CI:
$1.39-1.93 ; p<0.001)$. The strength of association persisted in all four multivariate models developed by adding covariates in a stepwise manner. In a multivariate model (model 4) in the matched sample which included all initial clinical and demographic variables, cardiovascular risk factors and eGFR, the presence of cancer
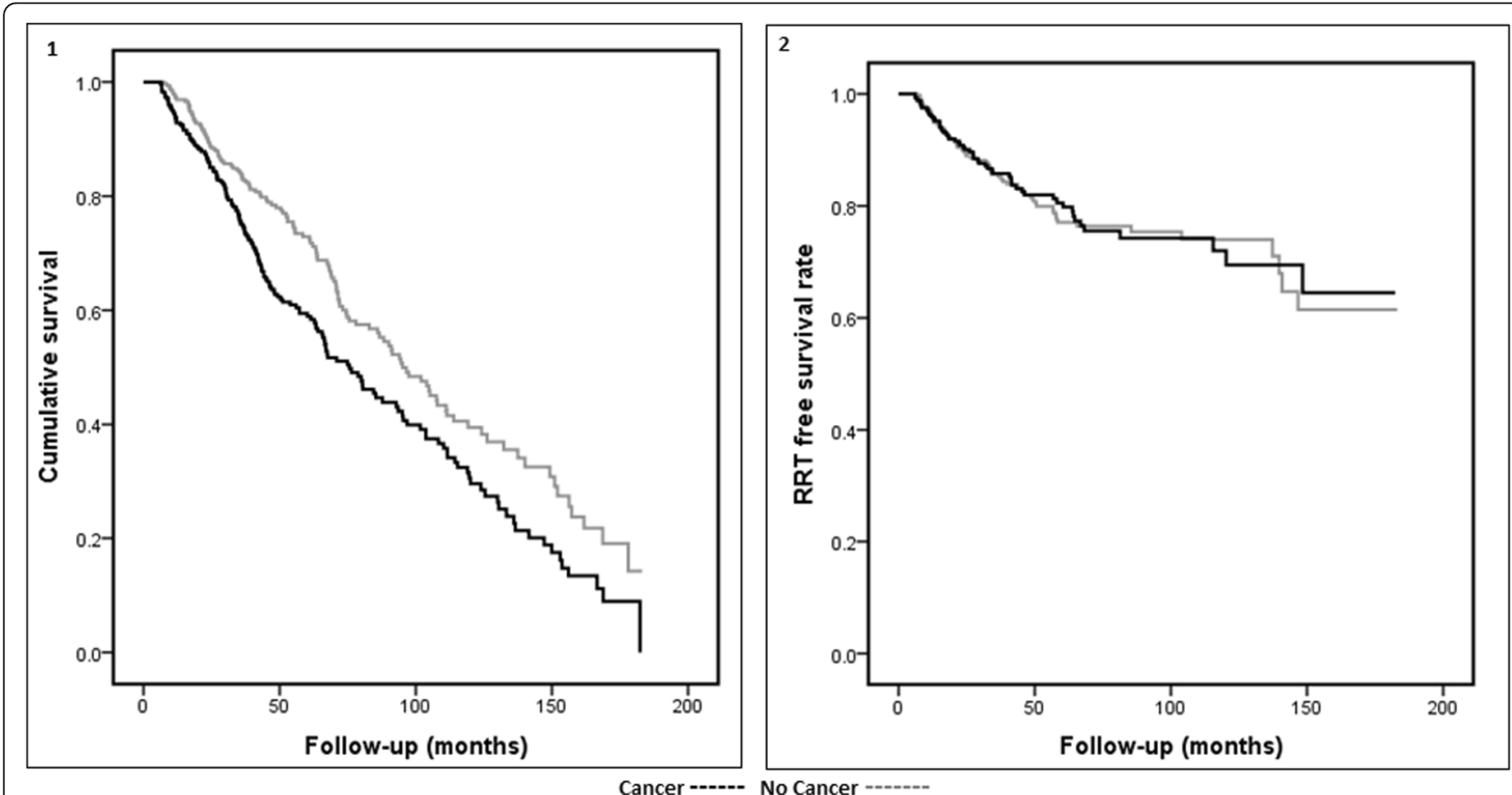

Fig. 3 Kaplan-Meier curve in the matched sample (panel-1: all-cause mortality, panel-2: renal replacement therapy (RRT) free survival) 
showed an independent association with all-cause mortality (HR: 1.41; 95\%CI: 1.12-1.78; $p=0.004$ ) (Table 3).

There was no clear correlation observed between cancer status and reaching end-stage renal disease (HR: 1.01; 95\%CI: 0.81-1.25; $p=0.97$ ) (Table 3). Also, CKD progression as determined by the rate of decline in eGFR was not different between the groups (cancer patients 0.96 versus $-1.24 \mathrm{~mL} / \mathrm{min} / 1.73 \mathrm{~m}^{2} /$ year) (Table 4 ). Similar observations were noted in the propensity matched sample with regards to the renal outcomes. In a subanalysis, urological cancers (kidney, bladder and prostate) were not associated with an increased risk of RRT either compared to non-cancer patients or those with cancer from other causes.

Competing risk models developed on the matched sample showed the probability of death was higher in the cancer group at 5, 10 and 15 years. At five years the probability of death was $36 \%$ in the cancer group compared to $23 \%$ in the no-cancer group, the difference being statistically significant $(p=0.001)$. There was no difference observed in the cumulative probability for renal replacement therapy or incident cancer between the groups (cancer vs no-cancer) during follow-up (Table 5) (Additional file 1: Figure S1).

The sub-analysis of 337 patients (matched sample) who had a previous or current cancer history at registration showed that 58 patients were in group A, 119 in group B and 160 in group C. A higher proportion of patients in group A (56.9\%) and B (56.3\%) died compared to that of group C (41\%) (A vs C, $p=0.04$ ). Death from cancer was more likely in group A $(35.3 \%$ vs 26.7 and $17.2 \%$ in groups $B$ and $C$, respectively) while death from cardiovascular disease was more prevalent in group $\mathrm{C}$ (34.5\%). RRT uptake was higher in group C (23\% vs $12.1 \%$ in group $\mathrm{A}$ ) with a greater proportion of ESRD patients receiving transplants in groups B and C (11.4 and $12 \%$ vs $5.3 \%$ in group A) (Table 6).

The KM analysis of these subgroups (A, B \& C) showed a distinction between the different cancer history and survival outcomes. As expected, patients with concurrent cancer at registration had the worst outcome (log-rank, $p$ Value $=0.001$ ) (Additional file 1: Figure S2). Although the KM graphs appear to be different between the groups for RRT free survival, this did not reach statistical significance (log-rank, $p$-Value $=0.079$; (Additional file 1: Figure S3). In a KM chart of outcomes of patients with different cancer sites in the matched sample there was an overlap of the survival pattern of certain cancer sites (breast/haematological) with the non-cancer group, but there was a clear distinction and poor survival noted for other cancer sites (lung, gastrointestinal tract, urogenital, other) (logrank, $p=0.001$ ) (Additional file 1: Figure S4).

\section{Discussion}

This is one of the largest studies examining cancer and its associations in a Caucasian cohort with advanced CKD. The study describes the pattern of cancer distribution in a nondialysis CKD population and its impact on mortality and renal outcomes. The concurrent or previous cancer history of $13.3 \%$ and annual incidence of $1.6 \%$ were similar to those observed in other CKD cohorts [33, 34]. Cancer site distribution was comparable to that seen in other CKD groups, with an understandably high prevalence of urological malignancy; the most prevalent and incident (35.7\%) cancers [35]. Surgical treatment of kidney and urothelial cancers would have led to the referral to nephrology services in many cases. However, the prevalence of liver cancer was noted to be low in our cohort $(0.26 \%)$, potentially reflecting a low prevalence of chronic liver disease $(2.9 \%)$ in the population.

Table 3 Association of cancer with all-cause mortality and end-stage renal disease (Cox regression models)

\begin{tabular}{|c|c|c|c|c|}
\hline & \multicolumn{2}{|l|}{ Total sample } & \multicolumn{2}{|c|}{ Propensity matched sample } \\
\hline & $\mathrm{HR}(95 \% \mathrm{Cl})$ & $p$-Value & $\mathrm{HR}(95 \% \mathrm{Cl})$ & $p$-Value \\
\hline \multicolumn{5}{|l|}{ All-cause mortality } \\
\hline Univariate model & $1.64(1.39-1.93)$ & $<0.001$ & $1.43(1.13-1.80)$ & 0.002 \\
\hline Multivariate model 1 & $1.40(1.19-1.66)$ & $<0.001$ & $1.37(1.09-1.73)$ & 0.006 \\
\hline Multivariate model 2 & $1.43(1.21-1.69)$ & $<0.001$ & $1.40(1.11-1.76)$ & 0.004 \\
\hline Multivariate model 3 & $1.49(1.26-1.76)$ & $<0.001$ & $1.40(1.12-1.77)$ & 0.004 \\
\hline Multivariate model 4 & $1.45(1.22-1.70)$ & $<0.001$ & $1.41(1.12-1.78)$ & 0.004 \\
\hline \multicolumn{5}{|l|}{ End-stage renal disease } \\
\hline Univariate model & $1.01(0.81-1.25)$ & 0.97 & $1.10(0.82-1.48)$ & 0.52 \\
\hline
\end{tabular}

Multivariate Model 1: Adjusted for age, gender, ethnicity

Multivariate Model 2: Adjusted for all covariates of model 1 plus smoking, alcohol, hypertension, diabetes mellitus

Multivariate Model 3: Adjusted for all covariates of model 2 plus ischaemic heart disease, myocardial infarction, congestive cardiac failure, cerebro vascular accident, peripheral vascular disease

Multivariate Model 4: Adjusted for all covariates of model 3 plus estimated glomerular filtration rate (CKD-EPI)

HR-Hazard ratio, Cl-Confidence interval. Statistically significant $\mathrm{p}$-values are displayed in bold (i.e. $p<0.05$ ) 
Table 4 Rate of decline of eGFR (CKD progression)

\begin{tabular}{|c|c|c|c|c|c|c|}
\hline \multirow[b]{2}{*}{ Variable } & \multicolumn{3}{|l|}{ Total sample } & \multirow{2}{*}{$\begin{array}{l}\text { Propensity matched } \\
\text { Cancer (328) }\end{array}$} & \multicolumn{2}{|l|}{ sample } \\
\hline & Cancer (332) & No cancer (2532) & $\overline{p \text {-Value }}$ & & No cancer (328) & $\overline{p \text {-Value }}$ \\
\hline eGFR slope $\mathrm{mL} / \mathrm{min} / 1.73 \mathrm{~m}^{2} /$ year & $-0.96(-2.8$ to 0.91$)$ & $-1.24(-3.2$ to 0.58$)$ & 0.07 & $-0.97(-2.9$ to 0.91$)$ & $-0.93(-2.71$ to 0.84$)$ & 0.93 \\
\hline
\end{tabular}

eGFR (CKD EPI)- estimated glomerular filtration rate calculated by CKD-EPI equation

Values expressed as median (interquartile range). $p$-Value by Mann-Whitney $U$ test

Rate calculated only from patients with minimum three eGFR results

In our analysis, patients with non-melanoma skin (NMS) cancers were included in the no cancer group as the cancer was localised in all patients, and all had curative treatment with no recurrence. The 10-year survival rate for NMS cancer with treatment is similar to people without cancer in the general population [36]. Patients in the cancer group were significantly older, an age-related association with cancer also noted in the general population [37]. It is well known that CKD is a pro-inflammatory state and chronic inflammation with ageing has been linked with tumorigenesis [38]. The CKD-EPI equation was used in the calculation of eGFR in our analysis due to the increasing evidence to support the use of this formula in cancer patients [39]. In the cancer group, median haemoglobin was marginally low and alkaline phosphatase was high, both of these observations probably a reflection of a lower eGFR ( $28.3 \mathrm{vs} 30 \mathrm{~mL} / \mathrm{min} ; p=0.01)$. However, these differences in baseline and biochemical characteristics were not observed once the groups were propensity matched.

In the matched samples, age at death was significantly lower in patients with cancer (79 versus 80.5 years, $p=$ $0.03)$. The KM estimate verified this difference in survival (log-rank; $p=0.001)$. All-cause mortality was high in cancer patients, predominantly influenced by more cancerspecific mortality $(26.3 \%$ versus $13.5 \%, p=0.005)$. The presence of CKD as comorbidity has been shown to be a risk factor for mortality in cancer patients [40]. Despite this, the cardiovascular disease (CVD) and infectionrelated mortality burden were similar in the two groups. It was also interesting to note that even in the cancer group

Table 5 Cumulative incidence probability for death and renal replacement therapy between the groups (cancer vs no cancer) in the matched sample in a competing risk analysis

\begin{tabular}{llll}
\hline Years from study entry & Groups & Death & Renal replacement therapy \\
\hline 5 & Cancer & 0.36 & 0.16 \\
& No cancer & 0.23 & 0.20 \\
10 & Cancer & 0.57 & 0.19 \\
& No cancer & 0.47 & 0.21 \\
15 & Cancer & 0.70 & 0.20 \\
& No cancer & 0.63 & 0.25 \\
& p-Value & $\mathbf{0 . 0 0 1}$ & 0.39
\end{tabular}

$p$-Value by modified $\mathrm{X}^{2}$ test by Gray. Statistically significant $\mathrm{p}$-values are displayed in bold (i.e. $p<0.05$ ) the CVD related mortality was equivalent to the cancerrelated mortality $(26.3 \%)$ and there was no significant difference in the number of NFCVE reported between the groups.

Both univariate and multivariate Cox-regression models have consistently shown the presence of cancer as a strong independent risk factor for all-cause mortality. In the model-3 of the multivariate Cox-regression analysis which was adjusted for baseline variables including all cardiovascular risk factors, cancer showed a HR: 1.49 (CI: 1.261.76; $p<0.001$ ) (Table 3). In the Japanese CKD cohort study of Tanaka et al. cancer was associated with cancerassociated but not all-cause mortality. However, our observations involved a considerably larger sample size than the Japanese study (2952 versus 515) [34].

The proportion of patients reaching ESRD were similar in the two groups. In the unmatched analysis, proportionately more cancer patients started haemodialysis with fewer transplants, but once propensity matching was undertaken these differences disappeared. There was no clear correlation between cancer status and reaching end-stage renal disease in the univariate model hence further multivariate models were not generated. Also, in linear regression analysis, the presence of cancer was not associated with accelerated rate of progression of CKD. The overall rate of decline in eGFR in our cohort was similar to that seen in other European cohorts [41]. The competing risk models inferred similar results; higher probability of death in the cancer group, with no difference in RRT uptake or incident cancer between the groups. To our knowledge this is the first study in the literature evaluating the association of baseline cancer status with renal outcome and progression in a nondialysis dependent European CKD cohort. It was evident from the subgroup analysis that survival outcome in patients with previous cancer was improved with a greater period of cancer in remission, inferring that these patients can be carefully considered for all RRT options including transplantation.

Our study does have some limitations not least the observational nature of the methodology. The missing cause of death data restricted our strength of conclusions in the matched sample due to small numbers. We were not able to account for cancer treatment status or stage at the time of recruitment into the cohort, a deficit which could introduce bias into the interpretation of the findings. As our study only included patients who 
Table 6 Comparison of outcomes between the groups split based on date of cancer occurrence prior to recruitment in matched sample

\begin{tabular}{llllll}
\hline Outcome & No cancer (337) & $\begin{array}{l}\text { Concurrent history of } \\
\text { cancer }<1 \text { year } \\
\text { Group A (58) }\end{array}$ & $\begin{array}{l}\text { Cancer history > 1 year and } \\
<5 \text { years } \\
\text { Group B (119) }\end{array}$ & $\begin{array}{l}\text { Cancer history }>5 \text { years } \\
\text { Group C (160) }\end{array}$ & $\begin{array}{l}p \text {-Value } \\
\text { Group A vs Group C }\end{array}$ \\
\hline Death & $129(38.3 \%)$ & $33(56.9 \%)$ & $67(56.3 \%)$ & $66(41.2 \%)$ & $\mathbf{0 . 0 4}$ \\
Cancer death & $6 / 52(11.5 \%)$ & $6 / 17(35.3 \%)$ & $8 / 30(26.7 \%)$ & $5 / 29(17.2 \%)$ & 0.16 \\
CVD death & $13 / 52(25 \%)$ & $2 / 17(11.8 \%)$ & $9 / 30(30 \%)$ & $10 / 29(34.5 \%)$ & 0.09 \\
ESRD & $87(25.8 \%)$ & $19(32.8 \%)$ & $24(20.1 \%)$ & $44(27.5 \%)$ & 0.45 \\
RRT & $65(19.3 \%)$ & $7(12.1 \%)$ & $14(11.8 \%)$ & $37(23.1 \%)$ & 0.07 \\
Transplants & $7 / 65(10.8 \%)$ & $0 / 7$ & $1 / 14(7.1 \%)$ & $2 / 37(7.4 \%)$ & $>0.05^{\mathrm{a}}$ \\
\hline
\end{tabular}

Cause of death data was available only in 52/129 of the no cancer group, 17/33 of group A, 30/67 of group B and 29/66 of group C. CVD cardiovascular disease, $E S R D$ end-stage renal disease, $R R T$ renal replacement therapy. $p$-Value by Chi-square test, a- $p$-Value by Fisher Exact test. Statistically significant $\mathrm{p}$-values are displayed in bold (i.e. $p<0.05)$

volunteered to participate in the cohort the generalisation of the age-adjusted cancer incident rates to the general CKD population is necessarily limited. Despite these, the study's strengths included a robust database with a large sample size, propensity matching and accurate follow-up data.

\section{Conclusions}

Our study has shown that cancer is a strong and independent risk factor for all-cause mortality in advanced CKD. Cardiovascular disease is still a leading cause of death in CKD patients, even in patients with cancer. Baseline cancer status did not accelerate the rate of progression of CKD. Our study findings imply that CKD patients with cancer have to be assessed on a case by case basis in planning for renal replacement therapy options. With advancing cancer management options, the presence of cancer should not be a limitation for RRT provision, including transplantation in selected cases.

\section{Supplementary information}

Supplementary information accompanies this paper at https://doi.org/10. 1186/s12882-019-1578-5.

Additional file 1: Figure S1. Cumulative incidence probability for death and renal replacement therapy (RRT) between the groups (cancer vs no cancer) in the matched sample. Figure S2. Kaplan-Meier curve for all-cause mortality in the matched sample (comparison between groups split based on date of cancer occurrence prior to recruitment). Figure S3. Kaplan-Meier curve for renal replacement therapy (RRT) free survival in the matched sample (comparison between groups split based on date of cancer occurrence prior to recruitment). Figure S4. Kaplan-Meier curve for all-cause mortality in the matched sample (comparison between groups split based on cancer sites with no cancer).

\section{Abbreviations}

BP: Blood pressure; Cl: Confidence interval; CKD: Chronic kidney disease; CRA: Competing risk analysis; CVD: Cardiovascular disease; eGFR: estimated glomerular filtration rate; EPR: Electronic patient records; ESRD: End-stage renal disease; HR: Hazard ratio; KM: Kaplan Meier; NFCVE: Non-fatal cardiovascular events; NMS: Non-melanomatous skin; RAS: Renin-angiotensin system; RRT: Renal replacement therapy; SKS: Salford Kidney Study

\section{Acknowledgements}

1. We extend our acknowledgements to all the research nurses involved in SKS data collection and management and all the study patients for consenting to participate in the study.

2. An abstract from the study was presented as a poster at the ERA-EDTA 2019 Congress.

\section{Authors' contributions}

Conception or design, or analysis and interpretation of data, or both-RC, EF, PK. Drafting the article or revising it -RC, GJ, PK. Providing intellectual content of critical importance to the work described- RC, GJ, PK. Final approval of the version to be published- RC, GJ, PK. All authors read and approved the final manuscript.

\section{Authors information}

RC: Senior Research Fellow (Renal Medicine), Salford Royal NHS Foundation Trust and University of Manchester.

GJ: Professor of Medical Oncology, University of Manchester; Consultant and Chair of Manchester Cancer Systemic Anti-Cancer Therapies Board, Ovarian Cancer, angiogenesis and drug development, The Christie NHS Foundation Trust, Manchester, UK.

PK: Professor of Nephrology, Salford Royal NHS Foundation Trust and University of Manchester; Academic Vice President, UK Renal Association; Chair, NIHR Renal Disorders national group, National Institute for Health Research.

Funding

No financial support.

\section{Availability of data and materials}

This study derives data from a precious long-standing database in which data has been meticulously collected over 20 years. The authors are shortly planning to perform further analyses from the data, and these would be compromised if the database were made publically available but are available from the corresponding author on reasonable request.

\section{Ethics approval and consent to participate}

Salford Kidney Study has ethical approval obtained from the North West Greater Manchester South Research Ethics Committee, UK (current REC reference: 15/NW/0818) and all patients involved in our observational analysis have signed an informed consent.

\section{Consent for publication}

Not applicable.

\section{Competing interests}

No competing interest to declare.

\section{Author details}

'Department of Renal Medicine, Salford Royal NHS Foundation Trust, M6 8HD, Salford, UK. Faculty of Biology, Medicine and Health, University of Manchester, Manchester, UK. ${ }^{3}$ Information Management and Technology, Salford Royal NHS Foundation Trust, Salford, UK. ${ }^{4}$ Manchester Cancer 
Research Centre, University of Manchester, Manchester, UK. ${ }^{5}$ The Christie NHS Foundation Trust, Manchester, UK.

\section{Received: 17 May 2019 Accepted: 9 October 2019} Published online: 22 October 2019

\section{References}

1. Wang H, Naghavi M, Allen C, Barber RM, Carter A, Casey DC, et al. Global, regional, and national life expectancy, all-cause mortality, and cause-specific mortality for 249 causes of death, 1980-2015: a systematic analysis for the global burden of disease study 2015. Lancet. 2016;388(10053):1459-544.

2. Mathers CD, Boerma T, Ma FD. Global and regional causes of death. Br Med Bull. 2009;92(1):7-32

3. Ferlay J, Colombet M, Soerjomataram I, Dyba T, Randi G, Bettio M, et al. Cancer incidence and mortality patterns in Europe: estimates for 40 countries and 25 major cancers in 2018. Eur J Cancer. 2018;103:356-87.

4. Weng S-F, Chiu Y-H, Jan R-L, Chen Y-C, Chien C-C, Wang J-J, et al. Death does matter-Cancer risk in patients with end-stage renal disease. Medicine (Baltimore). 2016;95(3):e2512.

5. Sankar D. Navaneethan, Jesse D. Schold, Susana Arrigain, Stacey E. jolly and JVN. Cause-specific deaths in non-Dialysis-dependent CKD. J Am Soc Nephrol. 2015;26:2512-20.

6. Sahni V, Choudhury D, Ahmed Z. Chemotherapy-associated renal dysfunction. Nat Rev Nephrol. 2009;5(8):450-62.

7. Wu M-Y, Chang T-C, Chao T-Y, Huang M-T, Lin H-W. Risk of colorectal Cancer in chronic kidney disease: a matched cohort study based on administrative data. Ann Surg Oncol. 2013;20(12):3885-91.

8. Chen J-S, Lu C-L, Huang L-C, Shen C-H, Chen SC-C. Chronic kidney disease is associated with upper tract Urothelial carcinoma. Medicine (Baltimore). 2016;95(14):e3255.

9. Park S, Lee S, Kim Y, Lee Y, Kang MW, Han K, et al. Risk of cancer in predialysis chronic kidney disease: a nationwide population-based study with a matched control group. Kidney Res Clin Pract. 2019;38(1):60-70.

10. Xu H, Matsushita K, Su G, Trevisan M, Ärnlöv J, Barany P, et al. Estimated glomerular filtration rate and the risk of Cancer. Clin J Am Soc Nephrol. 2019;14(4):530-9.

11. Lee $\mathrm{C}-\mathrm{H}$. Hepatocellular carcinoma in patients with chronic kidney disease. World J Gastroenterol. 2013;19(16):2466.

12. Na SY, Sung JY, Chang JH, Kim S, Lee HH, Park YH, et al. Chronic kidney disease in cancer patients: an independent predictor of cancer-specific mortality. Am J Nephrol. 2011;33(2):121-30.

13. Weng P-H, Hung K-Y, Huang H-L, Chen J-H, Sung P-K, Huang K-C. Cancerspecific mortality in chronic kidney disease: longitudinal follow-up of a large cohort. Clin J Am Soc Nephrol. 2011;6(5):1121-8.

14. Thompson S, James M, Wiebe N, Hemmelgarn B, Manns B, Klarenbach S, et al. Cause of death in patients with reduced kidney function. J Am Soc Nephrol. 2015;26(10):2504-11.

15. Izzedine H, Perazella MA. Onco-nephrology: an appraisal of the cancer and chronic kidney disease links. Nephrol Dial Transpl. 2015;30:1979-88.

16. Christensson A, Savage C, Sjoberg DD, Cronin AM, Frank O'Brien M, Lowrance $W$, et al. Association of cancer with moderately impaired renal function at baseline in a large, representative, population-based cohort followed for up to 30 years. Int J Cancer. 2013 Sep 15;133(6):1452-8.

17. Cosmai L, Porta C, Perazella MA, Launay-vacher V, Rosner MH. Opening an onconephrology clinic : recommendations and basic requirements. Nephrol Dial Transpl. 2018;33(July):1503-10.

18. de Vivar Chevez AR, Finke J, Bukowski R. The role of inflammation in kidney Cancer. Adv Exp Med Biol. 2014;816:197-234.

19. Coussens LM, Werb Z. Inflammation and cancer. Nature. 2002;420(6917): 860-7.

20. Stewart JH, Vajdic CM, Van Leeuwen MT, Amin J, Webster AC, Chapman JR, et al. The pattern of excess cancer in dialysis and transplantation. Nephrol Dial Transplant. 2009 Oct 1;24(10):3225-31.

21. Stewart JH, Buccianti G, Agodoa L, Gellert R, McCredie MR, Lowenfels AB, et al. Cancers of the kidney and urinary tract in patients on dialysis for endstage renal disease: analysis of data from the United States, Europe, and Australia and New Zealand. J Am Soc Nephrol. 2003 Jan 1;14(1):197-207.

22. Butler AM, Olshan AF, Kshirsagar AV, Edwards JK, Nielsen ME, Wheeler SB, et al. Cancer incidence among US Medicare ESRD patients receiving hemodialysis, 1996-2009. Am J Kidney Dis. 2015 May;65(5):763-72.
23. Mazzucotelli V, Piselli P, Verdirosi D, Cimaglia C, Cancarini G, Serraino D, et al. De novo cancer in patients on dialysis and after renal transplantation: North-Western Italy, 1997-2012. J Nephrol. 2017 Dec 19;30(6):851-7.

24. Alderson HV, Ritchie JP, Pagano S, Middleton RJ, Pruijm M, Vuilleumier N, et al. The associations of blood kidney injury Molecule-1 and neutrophil Gelatinase-associated Lipocalin with progression from CKD to ESRD. Clin J Am Soc Nephrol. 2016 Dec 7;11(12):2141-9.

25. Ritchie J, Rainone F, Green D, Alderson H, Chiu D, Middleton R, et al. Extreme elevations in blood pressure and all-cause mortality in a referred CKD population: results from the CRISIS study. Int J Hypertens. 2013;2013:1-8.

26. Gilg J, Rao A, Fogarty D. UK renal registry 15th annual report: chapter 1 UK RRT incidence in 2011: national and Centre-specific analyses. Nephron - Clin Pract. 2013;123(SUPPL. 1):1-28.

27. Levey AS, Stevens LA. Estimating GFR using the CKD epidemiology collaboration (CKD-EPI) Creatinine equation: more accurate GFR estimates, lower CKD prevalence estimates, and better risk predictions. Am J Kidney Dis. 2010;55(4):622-7.

28. Felix J. Thoemmes \& Eun Sook Kim. A systematic review of propensity score methods in the social sciences. Multivariate Behav Res. 2011:46(1):90-118.

29. Noordzij M, Leffondré K, Van Stralen KJ, Zoccali C, Dekker FW, Jager KJ. When do we need competing risks methods for survival analysis in nephrology? Nephrol Dial Transplant. 2013;28(11):2670-7.

30. R Development Core Team (2018), R: A Language and Environment for Statistical Computing. Vienna, Austria : the R Foundation for Statistical Computing. ISBN: 3-900051-07-0. Available online at https://cran.r-project. org/mirrors.html. (last accessed 15 April 2019).

31. Scrucca L, Santucci A, Aversa F. Regression modeling of competing risk using R: an in depth guide for clinicians. Bone Marrow Transplant. 2010; 45(9):1388-95.

32. Gray RJ. A class of k-sample tests for comparing the cumulative incidence of a competing risk. Ann Stat. 1988;16:1142-54.

33. Wong G, Staplin N, Emberson J, Baigent C, Turner R, Chalmers J, et al. Chronic kidney disease and the risk of cancer: an individual patient data meta-analysis of 32,057 participants from six prospective studies. BMC Cancer. 2016;16(1):1-11.

34. Tanaka A, Inaguma D, Watanabe $Y$, Murata $M$, Shinjo $H$, Koike $K$, et al. Relationship between mortality and Cancer-bearing status in patients with chronic kidney disease who attended an educational program. Ther Apher Dial. 2018;22(1):49-57.

35. Wong G, Zoungas S, Lo S, Chalmers J, Cass A, Neal B, et al. The risk of cancer in people with diabetes and chronic kidney disease. Nephrol Dial Transplant. 2012;27(8):3337-44.

36. Jensen $A \varnothing$, Lamberg $A L$, Jacobsen $J B$, Olesen $A B$, Sørensen HT. Nonmelanoma skin cancer and ten-year all-cause mortality: a population-based cohort study. Acta Derm Venereol. 2010;90(4):362-7.

37. White MC, Holman DM, Boehm JE, Peipins LA, Grossman M, Jane Henley S. Age and cancer risk: A potentially modifiable relationship. Am J Prev Med. 2014;46(3 SUPPL. 1):7-15.

38. Zinger A, Cho WC, Ben-Yehuda A. Cancer and aging - the inflammatory connection. Aging Dis. 2017;8(5):611-27.

39. Torres da Costa e Silva V, Costalonga EC, Coelho FO, Caires RA, Burdmann EA. Assessment of kidney function in patients with Cancer. Adv Chronic Kidney Dis 2018;25(1):49-56.

40. Wei Y-F, Chen J-Y, Lee H-S, Wu J-T, Hsu C-K, Hsu Y-C. Association of chronic kidney disease with mortality risk in patients with lung cancer: a nationwide Taiwan population-based cohort study. BMJ Open. 2018;8(1):e019661.

41. Brück K, Jager KJ, Zoccali C, Bello AK, Minutolo R, loannou K, et al. Different rates of progression and mortality in patients with chronic kidney disease at outpatient nephrology clinics across Europe. Kidney Int. 2018;93(6):1432-41.

\section{Publisher's Note}

Springer Nature remains neutral with regard to jurisdictional claims in published maps and institutional affiliations. 